\title{
Preliminary Study on the Role of TMEM39A Gene in Multiple Sclerosis
}

\author{
Marta Wagner $^{1}$ - Maciej Sobczyński ${ }^{2}$ Malgorzata Bilińska ${ }^{3} \cdot$ \\ Anna Pokryszko-Dragan ${ }^{3} \cdot$ Małgorzata Cyrul $^{3} \cdot$ Piotr Kuśnierczyk $^{1} \cdot$ Monika Jasek $^{1}$
}

Received: 4 April 2017 / Accepted: 13 April 2017 /Published online: 25 April 2017

(C) The Author(s) 2017. This article is an open access publication

\begin{abstract}
Genome-wide association studies (GWAS) have identified hundreds of new potential genetic risk loci associated with numerous complex diseases such as multiple sclerosis (MS). Genes which have been discovered by GWAS are now the focus of numerous ongoing studies. The goal of this study was to confirm and understand the potential role of one of such genes-transmembrane protein 39A gene (TMEM39A) - in multiple sclerosis.

We showed the difference in TMEM39A messenger RNA (mRNA) expression between MS patients and controls $\left(T_{2 ; 74}^{2}=5.429 ; p=0.0063\right)$. In our study, the lower mRNA expression of TMEM39A gene in patients did not correlate with a higher methylation level of the TMEM39A promoter. Moreover, a decreased level of TMEM39A mRNA was associated neither with rs1132200 nor with rs17281647. Additionally, we did not find an association between these two TMEM39A polymorphisms and the risk and progression of multiple sclerosis.
\end{abstract}

Electronic supplementary material The online version of this article (doi:10.1007/s12031-017-0921-1) contains supplementary material, which is available to authorized users.

Marta Wagner

marta_wagner@o2.pl; marta.wagner@iitd.pan.wroc.pl

$\bowtie$ Monika Jasek

jasek@iitd.pan.wroc.pl

1 Department of Clinical Immunology, Laboratory of Immunogenetics and Tissue Immunology, Hirszfeld Institute of Immunology and

Experimental Therapy, Polish Academy of Sciences, ul. Weigla 12, 53-114 Wrocław, Poland

2 Department of Genomics, Faculty of Biotechnology, University of Wrocław, ul. Fryderyka Joliot-Curie 14a, 50-383 Wrocław, Poland

3 Department and Clinic of Neurology, Wroclaw Medical University, ul. Borowska 213, 50-566 Wrocław, Poland
Our investigation is the first which indicates that TMEM39A mRNA expression may be associated with the development and/or course of multiple sclerosis.

Keywords Multiple sclerosis - TMEM39A - mRNA expression $\cdot$ Methylation $\cdot$ Polymorphism

\section{Introduction}

Multiple sclerosis (MS) is a chronic disease of the central nervous system leading to demyelination and axonal damage in the brain and the spinal cord (Pender and Greer 2007). It is estimated that the disease affects around 2.5 million people worldwide. The most common course of the disease (observed in about $85 \%$ patients) is relapsing-remitting (RR) MS, characterized by episodes of acute worsening followed by a recovery period. In the majority of the patients suffering from RR MS, the disease evolves into a secondary progressive (SP) phase characterized by a steady increase in disability without relapses. About $10-15 \%$ of patients experience primary progressive (PP) form of MS which is defined by the steady progression from the onset of the disease (Hoffmann and Meinl 2014; Inglese 2006).

Despite the ongoing studies, the etiology and pathogenesis of MS is still not well-defined. However, most scientists concur with the fact that the susceptibility to MS is determined by a combination of environmental and genetic factors. In recent years, thanks to the genome-wide association studies (GWAS), a large number of new genetic risk loci for MS such as IL7R, IL2RA, CLEC16A, CD58, EVI5, TYK2, etc. have been identified. Genes discovered by GWAS are now the focus of numerous ongoing studies which goal is to understand their potential role in MS susceptibility (International Multiple Sclerosis Genetics C 2010). 
In our study, we concentrated on the transmembrane protein 39A gene (TMEM39A), which was found to be associated with MS susceptibility in GWAS performed by International Multiple Sclerosis Genetics Consortium (IMSGC) (International Multiple Sclerosis Genetics C, 2010). Moreover, TMEM39A seems to be a susceptibility locus for systemic lupus erythematosus (Lessard et al. 2012; Sheng et al. 2015). Recent literature data suggest also that TMEM39A may serve as a novel diagnostic marker of gliomas and other cancers (Park et al. 2017). However, to date, very little is known about this gene and its function. Therefore, we decided to perform a preliminary functional study on TMEM39A and its role in MS. First, we investigated TMEM39A messenger RNA (mRNA) expression level in the group of 39 RR MS patients and 40 controls and then we studied TMEM39A methylation status. Moreover, we examined the effect of two TMEM39A polymorphisms (rs1132200 and rs17281647) on its mRNA level. Rs1132200 was indicated in literature as being associated with susceptibility to multiple sclerosis (International Multiple Sclerosis Genetics C, 2010) while rs17281647 polymorphism was selected on the basis of in silico analysis as Tag SNP that covers one of the linkage disequilibrium (LD) blocks of TMEM39A. Additionally, we conducted a case-control study (on 336 patients and 322 controls) to find a possible association of the investigated polymorphisms with susceptibility and/or progression of MS in a well-defined Polish population. Furthermore, in our study, we also took into account the presence of $H L A-D R B 1 * 15: 01$ allele.

\section{Materials and Methods}

\section{Study Population}

Peripheral blood samples from 39 RR MS patients (26 women and 13 men) with clinically definite MS according to the McDonald criteria (Polman et al. 2011) who did not have immunomodulatory therapy for at least 3 months were collected for mRNA isolation and TMEM39A expression analysis. Degree of disability and the rate of its progression were scored using Kurtzke's Expanded Disability Status Scale (EDSS) and MS Severity Score (MSSS), respectively (Kurtzke 1983; Roxburgh et al. 2005). The controls consisted of 40 volunteers ( 27 women and 13 men) with no history of inflammatory disease.

Methylation analysis and genotyping of two TMEM39A polymorphisms (performed in order to determine their influence on mRNA expression) were carried out on DNA samples from peripheral blood of the same 39 RR MS patients and 40 controls.

In the expanded study, concerning the association of TMEM39A polymorphisms with MS susceptibility and course of the disease, 336 patients (226 females and 110 males) were included. Two hundred fifty patients presented relapsing-remitting course of multiple sclerosis, while 86 had secondary progressive form of the disease. We did not include patients with primary progressive course in this study, since we did not collect sufficient number of samples to perform a powerful statistical analysis. Detailed patient characteristics are presented in our earlier publication (Wagner et al. 2014a). Controls were 322 blood donors (138 females and 184 males) with no history of inflammatory disease.

All the patients were under the charge of Department of Neurology, Wroclaw Medical University. The study was approved by the Commission of Bioethics at Wroclaw Medical University and written informed consent was obtained from all individual participants included in the study.

\section{RNA Isolation and mRNA Expression Analysis}

Whole blood samples were collected in PAXgene Blood RNA Tubes. Total RNA was extracted using the PAXgene Blood RNA Kit (Qiagen). The reverse transcription reaction of 500 ng of total RNA was carried out with iScript Reverse Transcription Kit (BioRad) according to the manufacturer's protocol.

mRNA expression was analyzed by qPCR using $5 \times$ HOT FIREPol EvaGreen qPCR Mix Plus (Solis BioDyne). Hypoxanthine phosphoribosyltransferase (HPRT) and glyceraldehyde 3-phosphate dehydrogenase (GAPDH) were used as reference genes. The primers used in this experiment are listed in Supplementary Table 1. Real-time PCR was performed in a final reaction mixture of $20 \mu \mathrm{l}$ containing $1.0 \mu \mathrm{l}$ of cDNA $(25 \mathrm{ng} / \mu \mathrm{l})$. The amplification conditions were as follows: $95{ }^{\circ} \mathrm{C}$ for $15 \mathrm{~min},\left(95^{\circ} \mathrm{C}\right.$ for $20 \mathrm{~s}, 61^{\circ} \mathrm{C}$ for $30 \mathrm{~s}, 72^{\circ} \mathrm{C}$ for $40 \mathrm{~s}) \times 40$ cycles. All samples were run in triplicate. In order to verify the reproducibility of results, the calibrator sample was run on each plate.

\section{Methylation Analysis-Methylation-Sensitive High-Resolution Melting (MS HRM)}

Genomic DNA was isolated from whole blood using Invisorb Blood Midi Kit (Stratec Molecular) according to the manufacturer's protocol.

EZ DNA Methylation-Gold Kit (Zymo Research) was used for bisulfite conversion of the DNA. The starting amount of DNA was $1 \mu \mathrm{g}$. All the modification reactions were performed according to the manufacturer's protocol.

PCR amplification followed by high-resolution melting analysis was carried out on ViiA7 Real-Time PCR System (Thermo Fisher Scientific). PCR was performed in a final reaction mixture of $20 \mu \mathrm{l}$ containing the following: $1 \times$ MeltDoctor HRM Master Mix, $250 \mathrm{nM}$ of each primer (please see Supplementary Table 1), $1.5 \mathrm{mM} \mathrm{MgCl}_{2}$, and 
$10 \mathrm{ng}$ of bisulfite-converted template. Primers were designed according to the protocol published by Wojdacz et al. (Wojdacz et al. 2008). The amplification conditions were as follows: $95{ }^{\circ} \mathrm{C}$ for $10 \mathrm{~min},\left(95{ }^{\circ} \mathrm{C}\right.$ for $15 \mathrm{~s}, 60{ }^{\circ} \mathrm{C}$ for $1 \mathrm{~min}) \times 40$. HRM analysis was performed using the following conditions: $95^{\circ} \mathrm{C}$ for $10 \mathrm{~min}, 60^{\circ} \mathrm{C}$ for $1 \mathrm{~min}, 95^{\circ} \mathrm{C}$ for $15 \mathrm{~s}$, and $60^{\circ} \mathrm{C}$ for $1 \mathrm{~s}$. All reactions were run in triplicate. Non-bisulfite-treated DNA was used as a negative control to ensure no amplification of genomic DNA. Methylation status of the examined samples was determined by comparing their melting profiles with the profiles derived from dilution series $(0,1,10,25,50,75$, and $100 \%)$ of the methylated and unmethylated control template (Human Methylated and Non-methylated DNA Set, Zymo Research). The normalized melting curves for each standard are shown in Supplementary Figure 1.

\section{Genotyping}

The examined SNPs of TMEM39A were genotyped by applying polymerase chain reaction followed by restriction fragment length polymorphism (PCR-RFLP). Supplementary Table 1 shows primer sequences, annealing temperatures, and restriction enzymes used in this study.

HLA-DRB $1 * 15: 01$ status was determined in our earlier studies (Wagner et al. 2013; Wisniewski et al. 2013) as described by Wisniewski et al. (Wisniewski et al. 2013), and these data were taken into account in the analyses performed here.

Accuracy of genotyping methods for the investigated SNPs was verified through direct sequencing of few samples representing homozygotes of two types and heterozygotes for each investigated SNP. These samples were used as the reference samples in following genotyping experiments.

\section{Statistical Methods}

TMEM39A mRNA expression (measured as $2^{-\Delta \mathrm{Ct}}$ ) normalized to $H P R T$ and to $G A P D H$ was described by median, 1 st (Q1), and 3rd (Q3) quartiles as well as by the minimal and maximal observation. In case of median, $S_{n}$ statistic was computed as the measure of variability: $S_{n}=\operatorname{med}\left\{\operatorname{med}\left|x_{i}-x_{j}\right| ; j=\right.$ $1 \ldots n\}$ (Rousseeuw and Croux 1993). $S_{n}$ is a typical difference between two randomly selected observations. Chisquare, $\chi^{2}$, test was used to test the hypothesis that two groups have the same distribution of genotype counts. When the sample sizes were small, distributions of the test statistics were estimated numerically. Odds ratio (OR) and confidence interval for them at $1-\alpha=0.95$ (CI 95\%) were computed as the measures of effect size. We assumed additive model of association between genotype and the risk of MS. TMEM39A mRNA expression, normalized to HPRT and GAPDH, was modeling as two-dimensional variable $x_{i}=\left(\mathrm{HPRT}_{i}\right.$, $\left.\mathrm{GAPDH}_{i}\right) \in \mathfrak{R}^{2}$ and significances was tested with $\lambda$ Pillai and
$T^{2}$ Hotelling statistics. Similarly, MS progression and its association with genetic factors was modeling as $x_{i}=\left(\mathrm{EDSS}_{i}\right.$, $\left.\operatorname{MSSS}_{i}\right) \in \mathfrak{R}^{2}$. Differences in the age of onset dependent on genetic factors were tested with analysis of variance F-statistic. Duration of the relapsing-remitting course was analyzed with the proportional hazards model. Haplotype frequencies were estimated with the maximum likelihood function (Excoffier and Slatkin 1995). Likelihood ratio statistic $\mathrm{LRS}_{d f=3}=2\left(L L_{\text {Cases }}+L L_{\text {Controls }}-L L_{\text {Combined }}\right)$ was used to test the differences in haplotype frequencies between cases and controls. Departure from Hardy-Weinberg equilibrium (HWE) was measured as $f=\frac{p_{C C}-p_{C}^{2}}{p_{C}\left(1-p_{C}\right)}$, where $p_{C}$ and $p_{C C}$ are allele $C$ and genotype $C C$ frequencies. $f<0$ in case of deficiency of homozygotes, $f>0$ corresponds to deficiency of heterozygotes, and $f=0$ when locus is in HWE. Linkage disequilibrium (LD) was measured with $r^{2}$ statistic. Results were considered as statistically significant at $p<0.05$.

\section{Results}

\section{TMEM39A mRNA Expression in Patients and Controls}

In the initial phase of our study, the level of TMEM39A mRNA expression in MS patients and controls was determined. We were able to observe a lower expression of TMEM39A mRNA in patients than in healthy individuals $\left(T_{2 ; 74}^{2}=5.429 ; p=0.0063\right)$ (Supplementary Table 2). Figure 1 shows TMEM39A mRNA expression normalized to two reference genes- $H P R T$ and GAPDH.

\section{Methylation Level of TMEM39A Gene}

Since methylation plays an important role in the regulation of gene expression and since in silico analysis revealed $\mathrm{CpG}$ islands in the promoter region of TMEM39A gene ( $\mathrm{Li}$ and Dahiya 2002), in the next step, we decided to check whether a lower expression of mRNA is correlated with a higher level of methylation of this gene. We were not able to find such relationship. In fact, we observed that TMEM39A gene was unmethylated both in controls and patients (Supplementary Figure 1B).

\section{TMEM39A mRNA Expression in Dependence of Polymorphisms rs17281647 and rs1132200}

Next, we wanted to find out whether the expression of TMEM39A mRNA is dependent on genotype at rs17281647 and rs1132200 polymorphic sites. We also took into consideration the presence of $H L A-D R B 1 * 15: 01$ allele (the most consistently replicated genetic risk factor for MS) and gender. The results showed that the analyzed variables were not 
Fig. 1 Difference in TMEM39A mRNA expression between MS patients and controls,

$T_{2 ; 74}^{2}=5.429 ; p=0.0063$. Thirtynine MS patients with relapsingremitting course of the disease and 40 controls were included in TMEM39A expression analysis. TMEM39A mRNA expression was normalized to $H P R T$ and $G A P D H$ and measured as $2^{-\triangle \mathrm{Ct}}$. Box-and-whiskers plot presents the median expression of TMEM39A gene in controls and patients, 1 st and 3rd quartiles, minimal and maximal non outlier observation

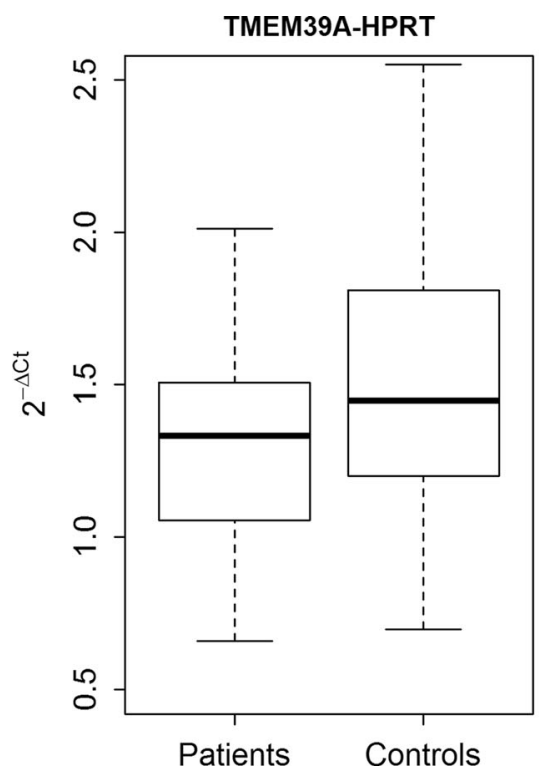

associated with TMEM39A mRNA expression (in combined group of patients and controls). Moreover, the lower expression of TMEM $39 \mathrm{~A}$ mRNA in MS patients was independent of these variables $\left(F_{\mathrm{df}=16 ; 138}=0.564 ; p=0.906\right)$.

\section{Polymorphisms vs MS Susceptibility and Clinical MS Data}

Additionally, we expanded the investigation of two TMEM39A polymorphisms (rs1132200 and rs17281647) in the group of 336 patients and 322 controls in order to find the possible association between these two SNPs and susceptibility and/or progression of MS.

For polymorphism rs17281647, we found no evidence for deviation from Hardy-Weinberg equilibrium both in controls and cases $\left(\chi^{2}=0.149 ; p=0.713\right.$ and $\chi^{2}=2.642 ; p=0.115$, respectively). Similarly, rs1132200 was in HWE in controls $\left(\chi^{2}=1.394 ; p=0.271\right)$, however in the patient group, deviation from HWE was observed $\left(\chi^{2}=6.14 ; p=0.021\right)$. In this group, we noticed the overrepresentation of GA heterozygotes $(f=-0.135$ CI $95 \%(-0.19 ;-0.06))$.

Our study revealed that neither of the SNPs analyzed by us was associated with MS risk in our population (Supplementary Table 3). Since in our case-control study $H L A-D R B 1 * 15: 01$ allele was strongly associated with MS (for $H L A-D R B 1 * 15: 01-/+\mathrm{OR}=2.66 ; 95 \% \mathrm{CI}=1.86-3.75$; $p<0.0001$ and for $H L A-D R B 1 * 15: 01+/+\mathrm{OR}=4.34 ; 95 \% \mathrm{CI}$ $=1.61-12.73 ; p=0.006$ ) (Wagner et al. 2014b) and stratification for $H L A-D R B 1 * 15: 01$ is expected to help to reveal the associations with MS susceptibility (Bronson et al. 2010; Kikuchi et al. 2002), the presence of this allele was also taken into consideration. However, the lack of association of genotypes in rs17281647 as well as in rs1132200 with susceptibility to multiple sclerosis was found in both $H L A$ -
$D R B 1 * 15: 01-$ and $H L A-D R B 1 * 15: 01+$ individuals (data not shown).

In the next phase, we analyzed the haplotype frequency in both the controls and the patients. We did not observe any differences in their distribution between the compared groups $\left(\mathrm{LL}_{\mathrm{df}=3}=6.308 ; p=0.098\right)$.

The analysis of linkage disequilibrium showed that the investigated SNPs were not in LD with each other in controls as well as in patients $\left(r^{2}=0.018\right.$ and $r^{2}=0.038$, respectively).

Since it was hypothesized that genes associated with susceptibility to MS (including TMEM39A) may also determine various features of its phenotype (Mowry et al. 2013), we examined the possible association between polymorphisms rs17281647 and rs1132200 and available clinical data. We observed that neither of them was associated with duration of relapsing-remitting course of the disease $\left(\chi_{\mathrm{df}=2}^{2}=1.764\right.$; $p=0.414)$. This result was adjusted to MSSS, EDSS, and age of onset, which were associated with duration of RR course of MS, as we showed in our previous study for ALCAM gene (Wagner et al. 2013). Furthermore, neither rs17281647 nor rs1132200 was associated with disease progression measured on the disability scales-EDSS and MSSS ( $\lambda_{\text {Pillai }}=0.013$; $p=0.377)$. We also found no evidence for association between TMEM39A polymorphisms and age of onset $\left(F_{\mathrm{df}=3 ; 222}=2.416 ; p=0.091\right)$.

\section{Discussion}

The results of the study performed by IMSGC, presenting TMEM39A (3q13.33) as potential MS susceptibility locus (International Multiple Sclerosis Genetics C 2010) prompted us to investigate this gene in well-defined Polish population. According to our knowledge, this is the first study to report 
evidence of different TMEM39A mRNA levels in MS patients and controls. Since, to date, very little is known about TMEM39A gene, it is hard to predict what role it may play with regard to multiple sclerosis. As stated in GeneCards database (www.genecards.org), TMEM39A may be involved in positive regulation of antiviral response by host. Since a large body of evidence indicates that infection with the EpsteinBarr virus has a major role in the pathogenesis of MS (Pender and Burrows, 2014), one might expect that lower expression of TMEM39A mRNA (assuming positive correlation between the levels of mRNA and protein) may be associated with higher susceptibility to infection with EpsteinBarr virus and as a consequence with higher susceptibility to multiple sclerosis. However, further studies are necessary to verify this hypothesis, to find out in what way lower expression of this gene may influence the susceptibility to MS and what mechanism is responsible for decreased TMEM39A mRNA expression.

One of the mechanisms which may cause silencing of the genes, possibly by blocking the binding of transcriptional factors to promoters, is DNA methylation. Recent evidence suggests the role of epigenetics (DNA methylation, histone modifications, and microRNA (miRNA)) in multiple sclerosis (Kucukali et al. 2015). Methylation status of several genes has been found to be altered in peripheral blood mononuclear cells (PBMC) and/or brain samples from MS patients as compared to those from healthy donors (Kucukali et al. 2015). The SHP-1 gene may serve as an example. The considerably reduced SHP-1 activity in PBMC of MS patients was noticed (Christophi et al. 2008) and it was associated with increased SHP-1 promoter methylation (Kumagai et al. 2012). This modification presumably results in increased proinflammatory gene expression and, as a consequence, in leukocyte-mediated inflammation (Kucukali et al. 2015). Taking the above into account, we decided to study the methylation level of TMEM39A promoter. Unfortunately, we were not able to observe higher methylation level correlated with lower TMEM39A expression in MS patients. In fact, we noticed that TMEM39A promoter was unmethylated both in controls and patients. It has to be mentioned that our study on the methylation of TMEM39A gene had preliminary status and further investigation of other putative functional methylation loci is necessary.

Taking into consideration the fact that altered mRNA expression may be caused by genetic variations, next, we decided to examine the potential influence of two TMEM39A polymorphisms (rs1132200 and rs17281647) on gene expression. Rs1132200 was indicated in the literature as being associated with susceptibility to multiple sclerosis (International Multiple Sclerosis Genetics C 2010; Varade et al. 2012) as well as systemic lupus erythematosus (Lessard et al. 2012; Sheng et al. 2015). This SNP causes non-synonymous amino acid change (Ala-Thr) at position 487 in the protein. However, it is not known, if it is a functional variant itself or if it is a marker for a different functional variant. The second polymorphism analyzed in our study, rs17281647, was selected on the basis of in silico analysis. In accordance with SNPinfo Web Server (Xu and Taylor 2009), rs17281647 is Tag SNP that covers one of the LD blocks of TMEM39A. Although rs17281647 is located in the intronic region, it is in strong LD with rs2282175 polymorphism located in the potential binding site for transcription factor, which may potentially alter the expression of TMEM39A. In our study, neither rs17281647 nor rs1132200 was associated with TMEM39A mRNA expression. On the one hand, lack of such association may be caused by the low number of samples analyzed in this study (since only patients who did not have immunomodulatory therapy for at least 3 months were taken into consideration). On the other hand, different (than those analyzed here) genetic variants or different mechanisms may underlie lower TMEM39A mRNA expression in MS patients.

Further studies are necessary to confirm lower expression of TMEM39A mRNA in MS patients and to find the mechanism which may be responsible for the decreased level of TMEM39A mRNA in MS patients. Since the expression of TMEM39A mRNA was measured in PBMC, it would be also essential to establish in further studies whether the lower level of TMEM39A mRNA is not the result of different proportion of subpopulation of cells expressing TMEM39A in samples from MS patients and controls and to check the correlation between mRNA and protein level.

Next, we decided to expand our study of TMEM39A polymorphisms into the group of 336 patients and 322 controls. We did not find an association of rs1132200 with MS susceptibility. In contrast to our findings, GWAS conducted by IMSGC (International Multiple Sclerosis Genetics C 2010) as well as replication studies performed on a Spanish population (Varade et al. 2012) and Indian population (D'Cunha et al. 2016) identified polymorphism rs $1132200 \mathrm{G}>\mathrm{A}$ of TMEM39A as the potential risk factor for MS with the common allele $(\mathrm{G}$ allele) increasing predisposition. According to Salanti et al. (Salanti et al. 2005), in the presence of an association, Hardy-Weinberg equilibrium in cases does not have to be maintained. Moreover, it was proposed that screening with HWE of data sets of affected individuals may be a relatively efficient method for detecting gene-disease associations (Salanti et al. 2005). Therefore, we presume that deviation from HWE observed by us in the group of patients $\left(\chi^{2}=6.14 ; p=0.021\right)$ may indicate the association of this polymorphism with MS also in our population and that the lack of significant difference in genotypes distribution between MS cases and controls may be caused by weak effect of this polymorphism on MS risk. Our study achieved $26 \%$ power to detect $\mathrm{OR} \leq 0.79$ (or equivalently $\mathrm{OR} \geq 1.26$ ) and $80 \%$ power to detect $\mathrm{OR} \leq 0.63$ (equivalently $\mathrm{OR} \geq 1.59$ ). It will be important to establish in follow-up studies on a larger 
population whether A allele in rs 1132200 is also the protective allele in the Polish population.

As we mentioned earlier, the second SNP analyzed here (rs17281647) is the intronic variant, however being in full LD $\left(r^{2}=1.0\right.$ according to SNPinfo Web Server) with polymorphism rs2282175 located in potential binding site for transcription factor. Moreover, the results of in silico analysis showed that it is also in full LD with rs1132202 SNP, which is located in the putative binding site for miRNA. We did not find the difference in genotype distribution in the analyzed polymorphic site between MS patients and healthy individuals. With our numbers of individuals in control and patient groups, we were able to detect $\mathrm{OR} \leq 0.82$ (equivalently $\mathrm{OR}$ $\geq 1.22$ ) with probability $P=0.23$ (power $23 \%$ ) and $\mathrm{OR} \leq 0.63$ (equivalently $\mathrm{OR} \geq 1.59$ ) with $P \geq 0.8$ (power $80 \%$ ). Since this is the first study which investigated the possible association of rs17281647 polymorphism with susceptibility to MS and course of the disease and because it achieved limited power to detect weak associations, further studies on a larger group are necessary.

Furthermore, we did not observe an association between rs1132200 and rs17281647 polymorphisms and progression of the disease.

In conclusion, although we were not able to find the association of TMEM39A polymorphisms with predisposition to multiple sclerosis and course of the disease, we showed that its mRNA expression is decreased in PBMC of MS patients.

Acknowledgments We wish to thank Izabela Nowak D.Sc. from the Laboratory of Immunogenetics and Tissue Immunology for great help in collecting DNA samples from healthy volunteers and Andrzej Wiśniewski PhD from the Laboratory of Immunogenetics and Tissue Immunology for providing part of $H L A-D R B 1 * 1501$ data. We are also very grateful to our patients and healthy volunteers for blood donation and agreement to participate in this study.

\section{Compliance with Ethical Standards}

Conflict of Interest The authors declare that they have no conflict of interest.

Open Access This article is distributed under the terms of the Creative Commons Attribution 4.0 International License (http:// creativecommons.org/licenses/by/4.0/), which permits unrestricted use, distribution, and reproduction in any medium, provided you give appropriate credit to the original author(s) and the source, provide a link to the Creative Commons license, and indicate if changes were made.

\section{References}

Bronson PG et al (2010) CIITA variation in the presence of HLA$D R B 1 * 1501$ increases risk for multiple sclerosis. Hum Mol Genet 19:2331-2340

Christophi GP et al (2008) SHP-1 deficiency and increased inflammatory gene expression in PBMCs of multiple sclerosis patients. Lab
Invest; J Tech Methods Pathol 88:243-255. doi:10.1038/labinvest. 3700720

D'Cunha MA, Pandit L, Malli C (2016) CD6 gene polymorphism rs17824933 is associated with multiple sclerosis in Indian population. Annals Ind Acad Neurol 19:491-494. doi:10.4103/0972-2327. 192384

Excoffier L, Slatkin M (1995) Maximum-likelihood estimation of molecular haplotype frequencies in a diploid population. Mol Biol Evol 12:921-927

Hoffmann F, Meinl E (2014) B cells in multiple sclerosis: good or bad guys?: an article for 28 May 2014 - World MS Day 2014. Eur J Immunol 44:1247-1250. doi:10.1002/eji.201470045

Inglese M (2006) Multiple sclerosis: new insights and trends AJNR. Am J Neuroradiol 27:954-957

International Multiple Sclerosis Genetics C (2010) Comprehensive follow-up of the first genome-wide association study of multiple sclerosis identifies KIF21B and TMEM39A as susceptibility loci. Hum Mol Genet 19:953-962. doi:10.1093/hmg/ddp542

Kikuchi S et al (2002) Estrogen receptor gene polymorphism and multiple sclerosis in Japanese patients: interaction with HLADRB1*1501 and disease modulation. J Neuroimmunol 128:77-81

Kucukali CI, Kurtuncu M, Coban A, Cebi M, Tuzun E (2015) Epigenetics of multiple sclerosis: an updated review. NeuroMolecular Med 17:83-96. doi:10.1007/s12017-014-8298-6

Kumagai C, Kalman B, Middleton FA, Vyshkina T, Massa PT (2012) Increased promoter methylation of the immune regulatory gene SHP-1 in leukocytes of multiple sclerosis subjects. J Neuroimmunol 246:51-57. doi:10.1016/j.jneuroim.2012.03.003

Kurtzke JF (1983) Rating neurologic impairment in multiple sclerosis: an expanded disability status scale (EDSS). Neurology 33:1444-1452

Lessard CJ et al (2012) Identification of IRF8, TMEM39A, and IKZF3ZPBP2 as susceptibility loci for systemic lupus erythematosus in a large-scale multiracial replication study. Am J Hum Genet 90:648660. doi:10.1016/j.ajhg.2012.02.023

Li LC, Dahiya R (2002) MethPrimer: designing primers for methylation PCRs. Bioinformatics 18:1427-1431

Mowry et al (2013) Association of multiple sclerosis susceptibility variants and early attack location in the CNS. PLoS One 8:e755565. doi: 10.1371/journal.pone.0075565

Park J et al (2017) Recognition of transmembrane protein 39A as a tumorspecific marker in brain tumor. Toxicological Research 33:63-69. doi:10.5487/TR2017.33.1.063

Pender MP, Burrows SR (2014) Epstein-Barr virus and multiple sclerosis: potential opportunities for immunotherapy. Clin Translat Immunol 3:e27. doi:10.1038/cti.2014.25

Pender MP, Greer JM (2007) Immunology of multiple sclerosis. Current Allergy Asthma Rep 7:285-292

Polman CH et al (2011) Diagnostic criteria for multiple sclerosis: 2010 revisions to the McDonald criteria. Ann Neurol 69:292-302. doi:10. 1002/ana.22366

Rousseeuw PJ, Croux C (1993) Alternatives to the median absolute deviation. JASA 88:1273-1283

Roxburgh RH et al (2005) Multiple sclerosis severity score: using disability and disease duration to rate disease severity. Neurology 64 : 1144-1151. doi:10.1212/01.WNL.0000156155.19270.F8

Salanti G, Amountza G, Ntzani EE, Ioannidis JP (2005) Hardy-Weinberg equilibrium in genetic association studies: an empirical evaluation of reporting, deviations, and power. Eur J Hum Genet 13(840). doi:10. 1038/sj.ejhg.5201410

Sheng YJ et al (2015) Association analyses confirm five susceptibility loci for systemic lupus erythematosus in the Han Chinese population. Arthritis Res Ther 17:85. doi:10.1186/s13075-015-0602-9

Varade J et al (2012) Replication study of 10 genes showing evidence for association with multiple sclerosis: validation of TMEM39A, IL12B and CBLB [correction of CLBL] genes. Mult Scler 18:959-965. doi: $10.1177 / 1352458511432741$ 
Wagner M, Wisniewski A, Bilinska M, Pokryszko-Dragan A, Nowak I, Kusnierczyk P, Jasek M (2013) ALCAM — novel multiple sclerosis locus interfering with HLA-DRB1*1501. J Neuroimmunol 258:7176. doi:10.1016/j.jneuroim.2013.02.015

Wagner M, Bilinska M, Pokryszko-Dragan A, Sobczynski M, Cyrul M, Kusnierczyk P, Jasek M (2014a) ALCAM and CD6 - multiple sclerosis risk factors Journal of neuroimmunology doi:10.1016/j. jneuroim.2014.08.621

Wagner M, Wisniewski A, Bilinska M, Pokryszko-Dragan A, Cyrul M, Kusnierczyk P, Jasek M (2014b) Investigation of gene-gene interactions between CD40 and CD40L in Polish multiple sclerosis patients Human immunology doi:10.1016/j.humimm.2014.05.013
Wisniewski A, Wagner M, Nowak I, Bilinska M, Pokryszko-Dragan A, Jasek M, Kusnierczyk P (2013) 6.7-kbp deletion in LILRA3 (ILT6) gene is associated with later onset of the multiple sclerosis in a Polish population. Hum Immunol 74:353-357. doi:10.1016/j. humimm.2012.12.006

Wojdacz TK, Dobrovic A, Hansen LL (2008) Methylation-sensitive high-resolution melting. Nat Protoc 3:1903-1908. doi:10.1038/ nprot.2008.191

Xu Z, Taylor JA (2009) SNPinfo: integrating GWAS and candidate gene information into functional SNP selection for genetic association studies. Nucleic Acids Res 37:W600-W605. doi:10.1093/nar/ gkp290 